\title{
EVALUACIÓN DE CARACTERÍSTICAS ESPECÍFICAS DE LA PERSONALIDAD EN DIFERENTES TRASTORNOS FÍSICOS
}

\author{
Gloria Díaz Acosta
}

(Recibido el 16/12/2006, aceptado el 18/04/2007)

\begin{abstract}
RESUMEN
Identifica los aportes de la investigación clínica sobre determinadas características de la personalidad en riesgo de enfermedad física, y que pueden ser evaluadas por instrumentos tipificados. Señala determinadas condiciones que favorece el abordaje para una comprensión integral del paciente.
\end{abstract}

Palabras clave: Evaluación clínica, Personalidad, Estrés psicológico, Depresión.

\begin{abstract}
It identifies the determined contributions of the clinical investigation on characteristic of the personlaity in risk of physical disease, and that can sert evaluated by diagnosed instruments. It indicates certain conditions that the boarding for an integral understanding of the patient favors.
\end{abstract}

Keywords: Clinical evaluation, Personality, Psychological stress depression.

En relación a la investigación sobre perfiles característicos de personalidad en diferentes trastornos físicos y el desarrollo de escalas periféricas para la detección de trastornos emocionales en diferentes patologías orgánicas, veremos aquellos en los que se ha desarrollado más ampliamente la investigación de características de personalidad, estos son: Los trastornos cardiovasculares y el cáncer:

a. Las enfermedades cardiovasculares son consideradas la principal causa de muerte en los países occidentales industrializados. La tasa de mortalidad cardiovascular se ha duplicado en los últimos veinte años.

Se ha logrado detectar una serie de factores de riesgo para la aparición de trastornos cardiovasculares (García Huete, 1991, 1993). Entre los factores considerados de alto riesgo, se encuentran tres principales: la hipertensión arterial (Donker, 1990) la hipercolesterolemia (Gluck, 1978) y el consumo de tabaco (Marston). Así como la diabetes, la obesidad, la edad. Y los factores de bajo riesgo, se consideran: el consumo de alcohol, los antecedentes familiares.

El estrés diario, insatisfacción laboral, la responsabilidad excesiva y numerosas situaciones vitales estresantes. 
Además se considera dos categorías de factores psicosociales de riesgo: (Bermudez y Cols, 1991):

- El estrés psicológico

Patrón de conducta tipo A (PCTA), identificado por Rosemman y Friedman, 1961, quienes la definieron como «un conjunto particular de acción-emoción que tiene cualquier persona comprometida en una lucha crónica para lograr un número de cosas normalmente ilimitadas de su ambiente, en el menor tiempo posible, y/o contra los esfuerzos opuestos de otras personas o cosas de su mismo ambiente».

- Algunas variables de personalidad

Las características principales que definen el PCTA son las siguientes:

a) Percepción de situaciones como amenazantes y competitivas

b) Búsqueda activa de tales situaciones

c) Gran afán por destacar siempre en todo lo que se lleva a cabo.

d) Realización (o intento de realización) de distintas tareas a la vez, con sensación de falta de tiempo y queja constante de ello.

e) Reacción agresiva y hostil ante las dificultades.

Desde el trabajo de Friedman y Rosenman se han realizado numerosas investigaciones acerca de la relación entre el PCTA y el desarrollo de enfermedades cardiovasculares: desarrollándose técnicas de evaluación precisa.

Destacándose las siguientes:

- Jenkis y Activity Survey (JAS, Escala de Actividad de Jenkins de Jenkis, Zuz < anski y Rosenman mide tres aspectos: implicación en el trabajo, impaciencia y competitividad).

- Jenkins Activity y Survery for Students (JASS, Escala de Actividad de Jenkins para estudiantes) de Krantz y Cols. Adaptación del JAS para población estudiantil.

b. La enfermedad oncológica, también es el campo de investigación e intervención de la psicología de la salud de gran importancia y actualidad.

El objetivo principal de los estudios realizados ha sido encontrar perfiles de personalidad que pronosticarán la posibilidad de padecer cáncer. De este modo se propuso el Patrón de Conducta Tipo C (PCTC), cuyas características son:

- Rasgos de personalidad tales como estoicismo, laboriosidad, perfeccionismo, sociabilidad, convencionalismo y controles defensivos rígidos.

- Dificultad para poder expresar emociones sobre todo las negativas tales como la ira.

- Tendencia a la depresión, entendida como desamparo y desesperanza. (Ibáñez, Romero y Andrew, 1992).

El estudio de las variables psicológicas relacionadas con el cáncer se ha orientado especialmente en el estudio de la relación entre cáncer y depresión; por lo que ésta sintomatología reviste gran interés: 
a) La depresión como variable premórbida. Stoll Solomon (1979), Eysenck (1983) e Ibáñez (1984) han mostrado como la presencia de trastornos depresivos está relacionada directamente con la aparición de déficit inmunológicos.

b) La depresión como reacción al diagnóstico y/o tratamiento del cáncer. Hinton (1963), Morris (1967), Meverowitz (1981), Barreto (1984) e Ibáñez (1990) encontraron que una cuarta parte de los pacientes tras recibir el diagnóstico, sufren depresión requiriendo de intervención directa y advirtieron de la posibilidad de 2 tipos de contaminación:

1. En los resultados: debido a que los datos estaban obtenidos en pacientes recién diagnosticados o en las primeras fases del tratamiento.

2. En los criterios: debido a que las pruebas utilizadas (Inventarios de Beck y de Hamilton) incluyen síntomas somáticos que pueden deberse al proceso oncológico.

Ibáñez (1991) recomienda el uso del inventario de depresión de Beck que es eficaz para la diferenciación de síntomas depresivos en pacientes severamente enfermos, así como la lista de síntomas de Stewart y Cols, que se mencionan a continuación:

1. Bajo estado de ánimo y pérdida de interés.

2. Al menos cuatro de los siguientes síntomas:

a. Dificultad de concentración

b. Irritabilidad

c. Sentimiento de devaluación

d. Miedo a «perder la cabeza»

e. Falta de iniciativa

f. Irrupciones de llanto

g. Pensamientos de suicidios

3. Retraimiento social

4. Anorexia, trastornos del sueño, fatiga y torpeza motora.

En cuanto a las técnicas de evaluación cabe señalar la dificultad de su adecuación para su utilización con pacientes oncológicos por interferencias de síntomas somáticos como cefaleas, pérdida de apetito que son derivados de trastornos psicológicos, pero estos síntomas son comunes en estos pacientes como consecuencia de la propia enfermedad y del tratamiento quimioterápico por lo que el psicólogo clínico y de la salud debe atender a los posibles efectos de la enfermedad en el conjunto de la personalidad, para no incurrir en falsos negativos en el diagnostico.

\section{EN CONCLUSIÓN}

La evaluación de la personalidad de los pacientes con trastorno físicos debe realizarse, bajo el contexto biopsicosocial, ya que la enfermedad es la ruptura del equilibrio de la organización jerárquica de la salud; en este contexto la salud es definida en términos del mantenimiento del equilibrio biopsicosocial. 
Al estar ligados el proceso de enfermar y la conducta de enfermedad a las estructuras de la personalidad el estudio de ésta, en el campo médico debe ser tomada de manera preponderante ya que las características psicológicas del paciente actúan como determinantes de los estilos de vida nocivos, en el comportamiento de los riesgos de las enfermedades, así como coadyuvan a la recuperación de la salud o genera interferencia en su recuperación.

En este sentido se considera tanto una evaluación global de las características de la personalidad, como una evaluación específica de la misma. Asimismo es necesario también tomar en cuenta una evaluación de la calidad de vida del paciente crónico encaminada a conseguir un nivel de bienestar y satisfacción vital de la persona afectada por su enfermedad.

Cabe mencionar que los pacientes con trastornos físicos, al tener características diferentes a los pacientes con enfermedades mentales, en su evaluación deben utilizarse instrumentos que cuenten con la respectiva validez y confiabilidad por existir correlatos psicológicos entre la enfermedad y la personalidad que deben conocerse. Por ende una evaluación realizada con instrumentos psicológicos, cuya validez está confirmada en sujetos con trastornos mentales no sería útil ni puede ser válida.

\section{REFERENCIAS BIBLIOGRÁFICAS}

Barreto y Bayés (1990). «El psicólogo ante el enfermo en situación terminal». Análisis de Psicología, 6 (2).

Barreto y Pascual (1991). La calidad de vida en el enfermo terminal. Universidad de Murcia. Bagés (1988). «Modulación psicológica de la respuesta inmunológica». Revista Cubana de Hematología.

Bayés (1991). Psicología oncológica. $2^{\mathrm{a}}$ ed., Barcelona.

Fernández Ballesteros (1994). Evaluación conductual hoy. Ed. Pirámide. Barcelona.

Ibáñez, E., Romero R., y Andreu, Y. (1992). «Personalidad tipo C. Una revisión crítica». Boletín de Psicología, 35, 49-79.

Ibáñez, E., (1984). «Reflexiones sobre las relaciones entre depresión y cáncer». Boletín de Psicología, 4, 489-795.

Ibáñez, E., (1990). «Estilos de vida y enfermedad: historia y vicisitudes de un concepto». En E. Ibáñez (dir.), Psicología de la salud y estilos de vida, Valencia, Promolibro, pp. 11-20.

Ibáñez, E., (1990). «Reflexiones sobre la relación entre depresión y cáncer». En E. Ibáñez (comps.): Psicología de la salud y estilos de vida, Valencia, Promolibro, pp. 39-52.

Pérez-García, A.M.; Sánchez-Elvira, A., Bermúdez, J. (1991). Hostilidad y el patrón de conducta tipo A: Análisis predictivo del JASE-H. II Semana de investigación de la Facultad de Psicología de la UNED, Madrid, mayo, pp. 6-11.

Pino, A. del y Gaos, M.T. (1991). Propiedades psicométricas de una versión española de la entrevista estructurada de Rosenman para la medida del PCTA. Barcelona.

Pino, A. del y Gaos, M.T. (1992). «La validez de la entrevista estructurada del PCTA», Boletín de Psicología. 35, 25-48.

Rodríguez Marín. J. (1993). «Evaluación en prevención y promoción de la salud», R. Fernández-Ballesteros, Evaluación conductual hoy. Un enfoque para el cambio en psicología clínica y de la salud, Madrid, Pirámide, pp. 652-714. 